\title{
Physicochemical study of Delphinium denudatum Wall (Ranunculales: Ranunculaceae) and their antioxidant activity
}

\author{
Anil Kumar Gupta ${ }^{1}$, , Mahfoozurrahman Khan ${ }^{2}$ and Danish Khan ${ }^{1}$ \\ ${ }^{1}$ Deptartment of Biotechnology. University of Mangalayatan. Campus Beswan. \\ Aligarh-202145. India. *Email: anilkumargupta0367@gmail.com. \\ ${ }^{2}$ Department of Applied Chemistry. Faculty of Engineering \& Technology. Aligarh \\ Muslim University, Aligarh, 202002, India.
}

\begin{abstract}
The drug jadwar known as Delphinium denudatum Wall (Ranunculales: Ranunculaceae), the jadwar consist of dried tuberous roots of $D$. denudatum. An annual glabrous or slightly downy herbs found is Western Himalayas from Kumaon to Kashmir at altitudes of $3,000 \mathrm{~m}$ to $4,500 \mathrm{~m}$ specially on grassy slopes. It is distinguished by its relatively small blue and violet color and it is one of the important drugs used as indigenous medicine in India, especially in where isolated from the petroleum ether-soluble fraction of roots of D. denudatum. Jadwar widely used in traditional unani system of medicine for the treatment of a variety of human ailments including epilepsy. In order to increase the bioavailability, the nanophytosome of the D. denudatum root aqueous fraction (DNP) was prepared, characterized and evaluation. The phospholipids complex of the obtain aqueous fraction (AF) was prepared with phospholipon $90 \mathrm{H}$. The size of nanophytosome was determined by dynamic light scattering. HPTLC fingerprinting of the $\mathrm{AF}$ was also performed.
\end{abstract}

Keywords: Delphinium denudatum; $\quad \beta$-sitosterol; Physicochemical properties; Antioxidant activity; Delphinium denudatum uses and applications; DPPH.

\section{Introduction}

The generic name Delphinium of jadwar is derived from a Greek word, which means dolphin. The word jadwar is Arabic from of Persian zadwar, which means the great purifier or antidote. It is externally covered by suberized metaderm, the metaderm comprises brown tubular cells having zoned what irregrated arrangement. Delphinium denudatum Wall (Ranunculales: 
Ranunculaceae) is used in ayurveda and unani, to treat cough, cold, non healing wounds effect. It is called as nirvisha in ayurveda. $D$. denudatum is also useful skin disorder, leucoderma etc.

D. denudatum (Figure 1) are extensively found in the Western Himalayas Kashmir at altitudes of 8,000 to 12,000 feet. Jadwar roots of $D$. denudatum wall is an important central nervous system active drug of unani system of medicine.

The mechanism of pharmacological action of $D$. denudatum roots can greatly be aided by the isolation of its active principle from the root and determination of the structurefunction relationship. Also, the potent curative effect of $D$. denudatum root extract against human ailments needs to be verified by controlled clinical studies. It has been mentioned to be redating, brain, and nerviness tonic. D. peregrium, $D$. staphisagria and $D$. triste are medicinally in Europen of which 15 species occur in India. It is used in treating asthma and respiratory disorders, useful in poisoning, antitoxic, gout, improves digestion strength, inflammation useful again snake bite, It has anti-convulsant activity.
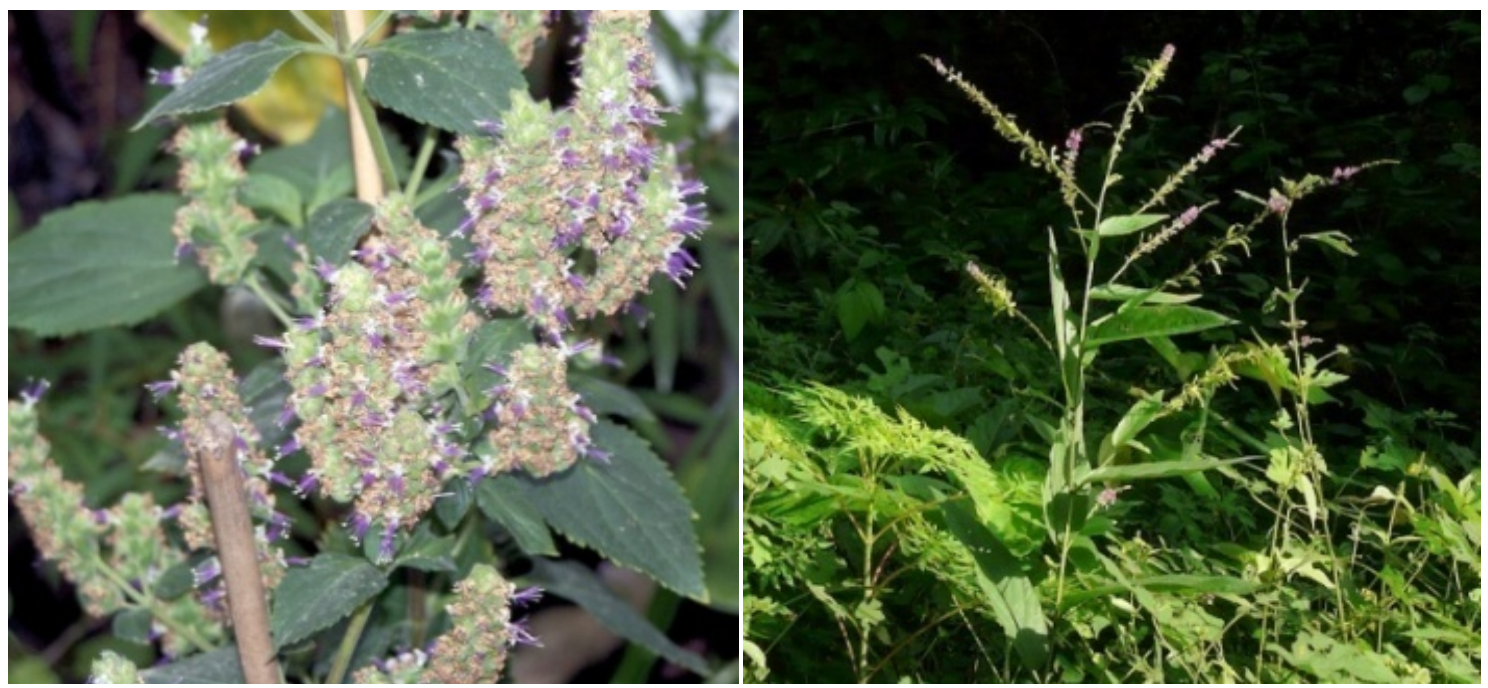

Figure 1. Delphinium denudatum Wall plants.

$\beta$-Sitosterol is one of several phytosterols (plant sterols) with chemical structure similar to that of cholesterol. Sitosterol are white, waxy powder with a characteristic order (Kamboj and Saluja, 2011). Theare hydrophobic and soluble in alcohols, $\beta$ Sitosterol a main dietary phytosterols found in plant, may have the potential for prevention and therapy for human cancer. Phytosterols are plant sterols found in food such as oils, nuts, and vegetable, phytosterols in same way as cholesterol, contain a double bond and are susceptible to oxidation and are characteristic by anti-carcinogenic and anti-atherogenic properties (Yoshida and Niki, 2003). It is one of the important drugs used as indigenous medicine in India, especially in unani.

Tamil: nirbasi nirvisi

Hindi: jadwar, judwar, nirbisi,

Marathi: nirvisha

$\beta$-sitosterol is unknown it may be related to cholesterol metabolism or 
anti-inflammatory effect. The present study was attempted to evaluate the antioxidant activity of the isolation compound from $D$. denudatum and to compare their antioxidant efficacy through DPPH method.

\section{Medicinal uses}

It is one of the important drugs used as indigenous medicine in India, especially in Unani medicine. The roots of the plant are reported to be useful in a variety of ailments such as aconite poisoning, brain diseases, fungal infection, piles and toothache as analgesic and astringent (Rawat and Jalal, 2011). Number of studies have been done on its phytochemical and pharmacological properties. Its use in opium addiction is mentioned in some classical literature, which has been verified and validated in morphine induced physical dependent de-addiction studies.

\section{Morphology}

D. denudatum (Figure 2) (root), dark brownish black in color, $3 \mathrm{~cm}$ to 6.5 $\mathrm{cm}$ long and $1 \mathrm{~cm}$ to $2 \mathrm{~cm}$ wide at the crown in length, conical shape, very hard, externally covered by a suberised metaderm, and bears numerous small circular scars. Outer region consisting of single layer of irregularly arranged brown tabular cell with subcrised walls; cortex consisting of narrow zone of about 5 to 10 layer thin walled, polygonal to rectangular parenchymatous cells. Flowers are about $2.5 \mathrm{~cm}$, with the upper outer petals about $1.2 \mathrm{~cm}$ and a spur 1.4$1.5 \mathrm{~cm}$. Upper inner petals are white, the others blue. Leaves are 5-15 cm across, rounded in outline, cut into 3-5 broadly obviate segments, segments pinnately cut into oblong lobes or teeth $2-3 \mathrm{~mm}$ wide.

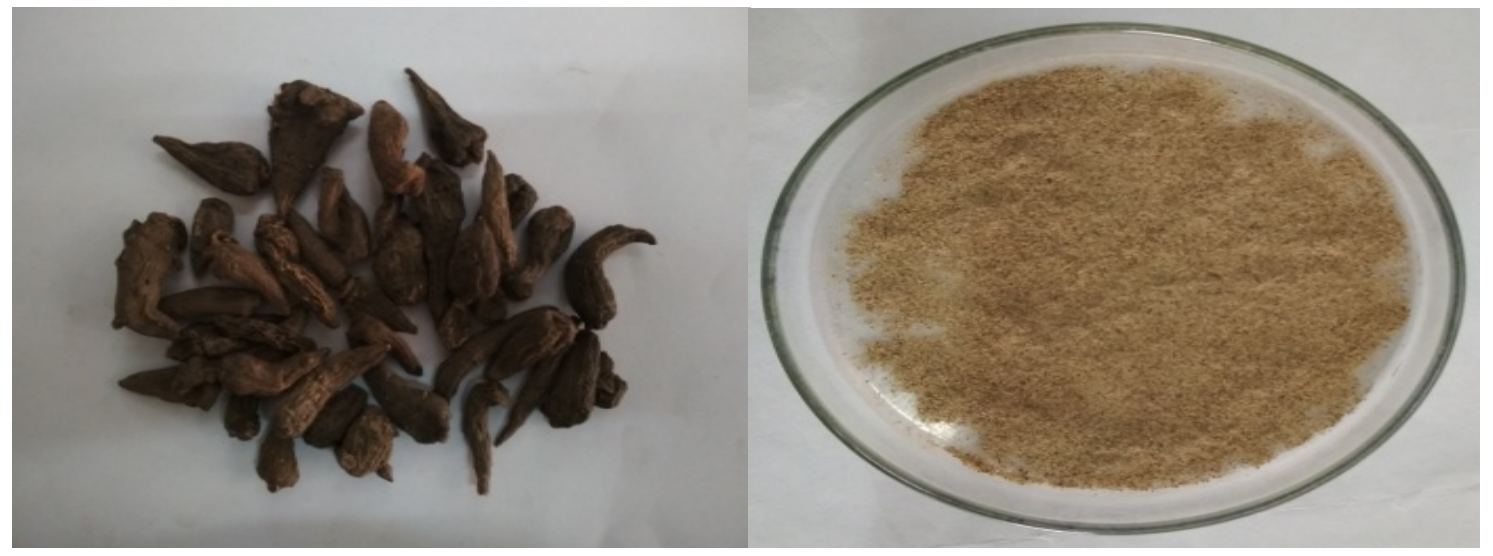

Figure 2. Rhizome (root) of Delphinium denudatum (Tamil-Nirbasi) and powder form.

\section{Physicochemical properties}

Physicochemical analysis is a method of investigating physicochemical system that make possible a determination of the nature of the interaction between the component of a system through study of the relations between the system physical properties and composition, the physicochemical study included the determination of extraction values of the test drugs in different solvents alcohol and water soluble contents, ash values, loss of weight on drying, bulk density, $\mathrm{pH}$ values. Analytical data of various physicochemical parameters indicates that some parameters like $\mathrm{pH}$, electrical conductivity, total dissolved solids, total 
suspended solids, turbidity and sodium are found to be in excess than the prescribed limit in some water samples of the study areas.

\section{Ash values}

$2.0056 \mathrm{~g}$ of drug was incinerated in a silica crucible of constant weight at a temperature not exceeding $450{ }^{\circ} \mathrm{C}$ in a muffle furnace until free from carbon fume after release the carbon fume cooled and weight of crucible from weight of crucible + ash.

\section{Water soluble ash}

The obtain Ash was boiled with $25 \mathrm{~mL}$ distilled water for $3 \mathrm{~min}$ to $5 \mathrm{~min}$. The insoluble matter was collected in an ash less filter paper (Whatman no. 42), washed with hot water and ignited in crucible, at temperature not more than $450{ }^{\circ} \mathrm{C}$.

The weight of insoluble Ash was subtracted from the weight of the total Ash, giving the weight of water soluble Ash. The percentage of water soluble Ash was calculated with reference to the air dried drug taken.

\section{Alcohol soluble ash}

The obtain ash was boiled with $25 \mathrm{~mL}$ Alcohol for $3 \mathrm{~min}$ to $5 \mathrm{~min}$. The insoluble matter was collected in an ash less filter paper (Whatman no. 42), washed with alcohol and ignited in crucible, at temperature not more than $450{ }^{\circ} \mathrm{C}$.

The weight of insoluble ash was of alcohol soluble ash was calculated with reference to the air dried drug taken. Subtracted from the weight of the total ash, giving the weight of alcohol soluble ash.

\section{Acid insoluble ash}

The total ash was boiled with $25 \mathrm{~mL}$ of $3 \mathrm{M}$ HCL solution of $10 \%$ for 5 min. The insoluble matter was collected on Ash less filter paper (Whatman no. 42), washed with Acid and ignited in crucible at a temperature not exceeding $450{ }^{\circ} \mathrm{C}$ the till constant weight.
The percentage of acid insoluble ash was calculated with reference to air dried drug taken. The acid insoluble ash content is the proportion of sample that is not hydrolyzed by $72 \%$ sulphuric acid and is not subsequently volatilized upon incineration of acid insoluble residue.

\section{Extractive values}

The extraction values of the drug in different solvent chloroform, ethyl acetate, alcohol and distilled water were determined by soxhlet method using a soxhlet apparatus. The heat was applied for six hours for each solvent on a heating montle. The extracts were filter and after evaporation of the solvent; the extractive values were determine with reference to the weight of drug. The procedure was repeated 3 times and the mean value for each extract was calculated.

\section{Loss on drying}

$1 \mathrm{~g}$ of drug was taken, spread uniformly and thin layered in a shallow petridis. It was heated at a regulated temperature of $105{ }^{\circ} \mathrm{C}$, cooled in desiccators and weighed. The process was repeated many times till two consecutive weights were found constant. The percentage of loss in weight was calculated with respect to initial weight.

\section{pH values}

Determination of $\mathrm{pH}$ was carried out by a Eutech digital pHmeter (Model no. 700), equipped with a combined electrode. The instrument was standardized by using buffer solution of 4.0, 7.0 and 10 to assertion the accuracy of the instrument prior to the experiment the $\mathrm{pH}$ values of $1 \%$ and $10 \%$ aqueous solution of powder drug solution was measured.

\section{Moisture content}

Karl Fischer (KF) titration is a technique for the determination of moisture content. It is a process based on the reaction of iodine with water. There 
are two methods used to perform the Karl Fischer titration test, volumetric and coulometric. Volumetric titration is used for moisture content above the 1\%-2\% range. The Karl Fischer method was used for the determination of moisture content. Before starting the titration, ensure the following things.

- Click the reagent bottle with the Karl Fischer reagent filled and tightly closed

Check solvent bottle with anhydrous methanol and tightly closed.

- Check the teflon tubes for their proper connection.

- Check the waste bottle(extreme right) clean and fitted with socket.

- Switch "ON" main power supply and "MAINS" of the instrument.

- Remove the Karl Fischer dispending tube and press clean key.

- $\quad$ Rinse the tip by pressing start key and fix it at its original position.

Presses start key.

- For the operating the instrument first neutralized the methanol by pressing start key. On neutralized it gives the beep sound and display show the volume consumed of Karl Fischer reagent.

- After neutralized transfer the sample in vessel and enter the weight of the sample and then press the start key and wait for the completion of the titration. The apparatus will calculate \& show the result automatically.

- Automatically calculation moisture content of sample with help of Karl Fischer is $0.71 \%$.

\section{(TLC)}

\section{Thin layer chromatography}

Thin layer chromatography (TLC) is a chromatography technique used to separate non-volatile mixtures. Thin layer chromatography is performed on a sheet of glass, plastic, or aluminium foil, which is coated with a thin layer of adsorbent material, usually silica gel, aluminium oxide (alumina), or cellulose. This layer of adsorbent is known as the stationary phase.

After the sample has been applied on the plate, a solvent or solvent mixture (known as the mobile phase) is drawn up the plate via capillary action. Because different analytes ascend the TLC plate at different rates, separation is achieved. The mobile phase has different properties from the stationary phase. For example, with silica gel, a very polar substance, non-polar mobile phases such as heptane are used. The mobile phase may be a mixture, allowing chemists to fine-tune the bulk properties of the mobile phase. Different compounds in the sample mixture travel at different rates due to the differences in their attraction to the stationary phase and because of differences in solubility in the solvent. By changing the solvent, or perhaps using a mixture, the separation of components (measured by the $\mathrm{R}_{F}$ value) can be adjusted. Also, the separation achieved with a TLC plate can be used to estimate the separation of a flash chromatography column.

Extract $2 \mathrm{~g}$ of sample with $10 \mathrm{~mL}$ chloroform under reflex on a water bath for $25 \mathrm{~min}$ to $30 \mathrm{~min}$. Filter and concentrate to $5 \mathrm{~mL}$ and carry out the thin layer chromatography.

A. Apply the chloroform and extract on TLC plate (Figure 3). Develop the plate to distance of $8.2 \mathrm{~cm}$ using Toluene: Ethyl Acetate $(5: 1.5)$ as mobile phase. After development allow the plate to dry in air and examine under U.V. (366 nm). It show major or spot at $\mathrm{R}_{\mathrm{F}} 0.85$ (sky blue), 0.72 (light blue). Dip the plate in Vanillin-Sulphuric Acid reagent followed by heating $100{ }^{\circ} \mathrm{C}$ to $115{ }^{\circ} \mathrm{C}$ for about $5 \mathrm{~min}$ to $10 \mathrm{~min}$ and observe under visible light. The plate show major spots at $\mathrm{R}_{\mathrm{F}} 0.9$ (dark blue), 0.07 (violet) 0.63 (dark blue). 


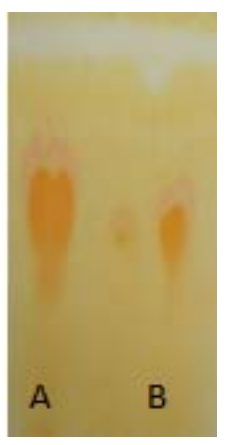

Figure 3. TLC (chloroform) of rhizome (root) of Delphinium denudatum (Tamil-Nirbasi) (A) (Standard Sample of Delphinium denudatum) (B) (Delphinium denudatum sample)

B. Apply the alcohol and on extract on TLC plate (Figure 4). Develop the plate to distance of $8.2 \mathrm{~cm}$ using Toluene: Ethyl Acetate $(5: 1.5)$ as mobile phase. After development allow the plate to dry in air and examine under U.V. (366 nm). It show major or spot at $\mathrm{R}_{\mathrm{F}} 0.88$ (sky blue), 0.086 (light blue). Dip the plate in Vanillin-Sulphuric acid reagent followed by heating $100{ }^{\circ} \mathrm{C}$ to $115{ }^{\circ} \mathrm{C}$ for about $5 \mathrm{~min}$ to $10 \mathrm{~min}$ and observe under visible light. The plate show major spots at $\mathrm{R}_{\mathrm{F}} 0.98$ (dark blue), 0.13 (violet).

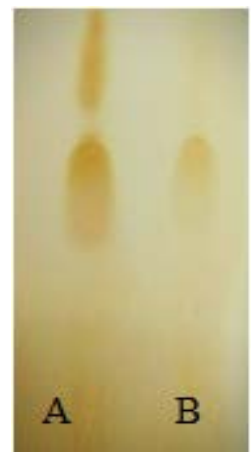

Figure 4. TLC (alcohol) of rhizome (root) of Delphinium denudatum (Tamil-Nirbasi) (A) (Standard Delphinium denudatum), Sample of (B) (Delphinium denudatum sample).

Table 1. Physicochemical study of powder of Delphinium denudatum Wall.

\begin{tabular}{llc}
\hline S. No. & Parameter & Percentage(w/w) \\
\hline $\mathbf{1}$ & Ash values & 2.16 \\
& Total ash & 0.36 \\
& Acid insoluble ash & 46.85 \\
\hline $\mathbf{2}$ & Water soluble ash & \\
& Soluble part & 4.85 \\
& Ethanol soluble & 6.79 \\
\hline $\mathbf{3}$ & Aqueous soluble & \\
& Successive extractive values & 2.4 \\
& Chloroform & 6.85 \\
& Alcohol & 14.21 \\
\hline $\mathbf{4}$ & Ethyl Acetate & 1.18 \\
\hline $\mathbf{5}$ & Distilled Water & 5.26 \\
& Loss on drying & 5.45 \\
\hline & pH values & 0.43 \\
\hline
\end{tabular}




\section{Materials and methods} sample

Collection of plant rhizome

Rhizome (root) of D. denudatum (Tamil-Nirbasi) was purchased from Khari Baoli Market, Old Delhi, India. The sample was authenticated by Prof. Sagheer Ahmed, R\&D Head of Department Hamdard Laboratory Ghaziabad.

Preparation of crude extract of

\section{D. denudatum rhizome}

The root sample $(D$. denudatumrhizome) was washed well and shadedried. Rhizome were chopped into small pieces and grounded to a fine powder by using grinder mixer. The solvent petroleum ether was used for the crude extracts preparation. The powered root material was packed in the filter paper and placed gently in the soxhlet apparatus. The extraction was carried out for $14-16$ cycles at $50{ }^{\circ} \mathrm{C}$. The crude extract obtained was further concentrated by using rotary evaporator and used for the further studies.

\section{Isolation of $\beta$-Sitosterol from $D$.}

\section{denudatum rhizome}

The petroleum ether extract of $D$. denudatum root was subjected to column chromatography on silica gel $(60-120$ mesh size), which was eluted with hexane, hexane-ethyl acetate, ethyl acetate mixtures inincreasing order of polarity to yield fine major fractionsnamely Fraction A (hexane), Fraction B (hexane-ethylacetate; 95:5), Fraction C (hexane-ethyl acetate; 90:10), Fraction D (hexane-ethyl acetate; 85:15) and Fraction E (ethyl acetate) (Patra et al., 2010). The eluted fractions were analyzed forthe presence of the sterol. The individual fraction were spotted on the TLC plates using capillary tube and separated using hexane/ethyl acetate (7:3.1) mobile phase. The plates were developed by dipping them in methanolic $\mathrm{H}_{2} \mathrm{SO}_{4}$ reagent for few sec and are placed in the hot air oven at $60^{\circ} \mathrm{C}$ for 4-6 min.

Appearance of pink color bands on the TLC plates indicates the zones of steroidal nucleus. The fraction $B$ obtained from hexane-ethyl acetate (95:5) were purified by chromatography using silica gel (200-400 mesh size) eluting with hexane-ethyl acetate (89:10.1) to obtain two sub-fractions F1 and F2. The sub-fraction were treated with methanolic $\mathrm{H}_{2} \mathrm{SO}_{4}$ reagent, the appearance of pink color band on the F1 sub-fraction on the TLC plates indicates the presence of sterol. The structural details ofthe isolated compound obtained from the fraction B (sub-fraction F1) were analyzed based on the elemental analysis and spectroscopic evidence (UVVis) spectroscopy.

The activity of scavenging (\%) was calculated using the following equation: DPPH radical scavenging:

\section{$(\%)=[(O D$ control - OD sample $) / O D$ control $] \times 100$}

\section{Results}

Characterization of herbal drug is essential for the quality control to check the presence of adulterants as a single drug remedy or its polyherbal unani formulation.

From the positive test for steroids by methanolic $\mathrm{H}_{2} \mathrm{SO}_{4}$ reagent, it is assumed to be a fraction $\mathrm{B}$ which showed clear band with less impurities and pink color band indicating the presence of sterol constituents. Fraction $B$ was further purified to yield subfraction F1 by column chromatography using the same solvent system hexane: ethyl acetate (89:10.1) and finally obtained pure single compound. The purity of the isolated compound was analyzed by thin layer chromatography 
(Figure 5). The spot on the TLC chromatogram developed with hexaneethyl acetate gave a positive result in Libermann-Burrchad test and methanolic $\mathrm{H}_{2} \mathrm{SO}_{4}$ reagent indicating a steroidal substance. Preliminary identification of the compound was based on the comparison of authentic standard compound. The melting point of the isolated compound was found to $136^{\circ} \mathrm{C}-136.3^{\circ} \mathrm{C}$.

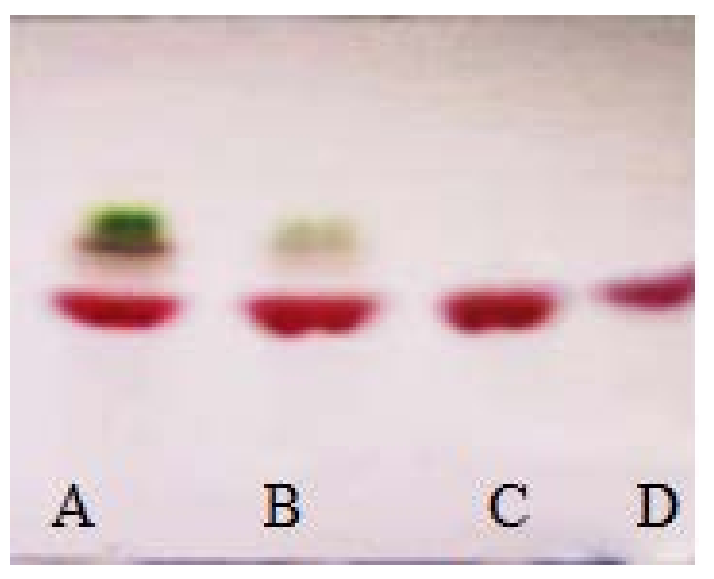

Figure 5. Chromatogram of fraction B, fraction $\mathrm{F} 1$ and isolated compound compared with standard ( $\beta$-Sitosterol) TLC. A-Fraction B eluted with hexane; ethyl acetate (95: 5); BSub-fraction F1 eluted with hexane, ethyl acetate (89: 10.1); C-Isolated compound; DStandard ( $\beta$ - Sitosterol).

\section{Discussion}

The present study is an attempt to ascertain the pharmacopoeial standard for the standardization of the $D$. denudatum sample the purity, quality, identity and strength of the powder has been undertaken as a tool to bring out several features like ash standards, solubility in alcohol and water, successive extraction values, and qualitative screening of physicochemical studies (Anonymous, 1952; India, 2010; Rahman et al. 1997).

Bioactive compound are found in both plant and animal products or can be synthetically produced. Example of plant bioactive compound is carotenoids and polyphenols. A bioactive compound is that an effect on a living organism, tissue/cell (Mohanapriya and Siva, 2013). After a plant has identified as a source for new product, bioactive compound are located in the relevant fraction using so called "Bioassay guided isolation". In this process the plant are successively extracted with solvents of increasing polarity and tested by a range of bioassays relevant to the activity of the compound one is trying to isolation by using the relevant enzyme system, micro-organisms, organs, etc. Plants contain many bioactive compounds that are nonnutritive plant chemicals rendering defense mechanism to plants. These compound also posse's properties that could act as antimicrobial, antioxidant and anticancer agents so the isolation and study of the groups of compound may help to demonstrate their specific properties. Spraying of $10 \%$ methanolic sulfuric acid reagent on TLC plate developed the pink color bands indicate the presence of sterol and similar reports were documented by Wagner and Bladt (1996). The spectroscopic evidence obtained in the present study was comparable to the earlier reports. Spectroscopic technique is one of the most powerful and analytical tool for the qualitative and quantitative analysis of bioactive compound.

$\beta$-Sitosterol can scavenge the radicals generated by DPPH method by donating their hydrogen atom to quell the free radicals, indicating that $\beta$ Sitosterol has potential antioxidant properties. Furthermore, other reports have shown a significant free radical scavenging capacity of $\beta$-Sitosterol. When the compound was examined in plant extracts (Patra et al. 2010).

\section{Conclusion}

Physicochemical standardization is of prime importance in quality control 
of unani drugs. As the efficacy of many drugs mainly depends upon its physical and chemical properties therefore, the determination of physical-chemical characters for the authenticity of drug is necessary before studying any medicinal property.

The results of the present holistic approach towards the identification and isolation of the bioactive constituent and antioxidant efficacy of $\beta$-Sitosterol from the extract of $D$. denudatum showed a highly active toward DPPH free radical scavenging assay. The ability of the sample to scavenge DPPH radical was determined from:

\section{DPPH radical scavenging $(\%)=[(O D$ control-OD sample $) / O D$ control $] \times 100$}

\section{Conflict of interests}

The authors declare that there are no conflicts of interest.

\section{References}

Anonymous. The wealth of India. Raw materials. New Delhi: CSIR: 1952.v. 3.

India. Unani pharmacopoeia of India. New Delhi: Department of Ayush, 2009. v. 6.

Kamboj, A.; Saluja, A. K. Isolation of stigma sterol and $\beta$-sitosterol from petroleum ether extract of aerial parts of Ageratum conyzoides (Asteraceae). International Journal of Pharmacy and Pharmaceutical Sciences, v. 3, p. 94-96, 2011.

Mohanapriya, S.; Siva, G. V. Antioxidant and bioactive constituent and its compounds. Journal of Nutritional Science and Vitaminology, v. 2, p. 138-141, 2013.

Patra, A.; Jha, S.; Murthy, P. N.; Manik; Sharone, A. Isolation and characterization of stigmast-5-en-3 $\beta$-ol ( $\beta$-Sitosterol) from the leaves of Hygrophila spinosa T. Anders. International Journal of Pharmaceutical Sciences and Research, v. 1, p. 95-100, 2010.
Rahman, A.; Nasreen, A.; Akhtar, F.; Shekhani, M. S.; Clardy, J.; Parvez, M.; Choudhary, M. I. Antifungal diterpenoid alkaloids from Delphinium denudatum Wall. Journal of Natural Products, v. 60 , no. 5, p. 472-474, 1997.

Rawat, V. S.; Jalal, J. S. Sustainable utilization of medicinal plants by local community of Uttarkashi District of Garhwal, Himalaya, India. European Journal of Medicinal Plants, v. 1, no. 2, p. 18-25, 2011.

Wagner, H.; Bladt, S. Plant drug analysis: A thin layer chromatography Atlas. 2. ed. New York: Springer-Verlag, 1996.

Yoshida, Y.; Niki, E. Antioxidant effects of phytosterols and its components. Journal of Nutritional Science and Vitaminology, v. 49, p. 277-280, 2003.

License information: This is an open-access article distributed under the terms of the Creative Commons Attribution License, which permits unrestricted use, distribution, and reproduction in any medium, provided the original work is properly cited. 